\title{
Antishadowing Effect on Charmonium Production at a Fixed-Target Experiment Using LHC Beams
}

\author{
Kai Zhou, ${ }^{1,2}{\text { Zhengyu Chen, }{ }^{1} \text { and Pengfei Zhuang }}^{1}$ \\ ${ }^{1}$ Physics Department, Tsinghua University and Collaborative Innovation Center of Quantum Matter, Beijing 100084, China \\ ${ }^{2}$ Institute for Theoretical Physics, Goethe-University Frankfurt am Main, Max-von-Laue-Strasse 1, \\ 60438 Frankfurt am Main, Germany
}

Correspondence should be addressed to Pengfei Zhuang; zhuangpf@mail.tsinghua.edu.cn

Received 17 April 2015; Revised 25 June 2015; Accepted 5 August 2015

Academic Editor: Cynthia Hadjidakis

Copyright (C) 2015 Kai Zhou et al. This is an open access article distributed under the Creative Commons Attribution License, which permits unrestricted use, distribution, and reproduction in any medium, provided the original work is properly cited. The publication of this article was funded by SCOAP S $^{3}$

We investigate charmonium production in $\mathrm{Pb}+\mathrm{Pb}$ collisions at LHC beam energy $E_{\mathrm{lab}}=2.76 \mathrm{ATeV}$ at fixed-target experiment $\left(\sqrt{s_{\mathrm{NN}}}=72 \mathrm{GeV}\right)$. In the frame of a transport approach including cold and hot nuclear matter effects on charmonium evolution, we focus on the antishadowing effect on the nuclear modification factors $R_{\mathrm{AA}}$ and $r_{\mathrm{AA}}$ for the $J / \psi$ yield and transverse momentum. The yield is more suppressed at less forward rapidity $\left(y_{\mathrm{lab}} \simeq 2\right)$ than that at very forward rapidity $\left(y_{\mathrm{lab}} \simeq 4\right)$ due to the shadowing and antishadowing in different rapidity bins.

\section{Introduction}

Recently a fixed-target experiment using the LHC beams has been proposed [1], where the study on quarkonia in nuclear collisions becomes specifically important, due to the wide parton distributions in phase space which is helpful to reveal the charmonium production mechanism [2]. Corresponding to the LHC beam energy $E_{\mathrm{lab}}=2.76 \mathrm{ATeV}$, where $A$ is the nucleon number of the incident nucleus, the center-of-mass energy $\sqrt{s_{\mathrm{NN}}}=72 \mathrm{GeV}$ is in between the SPS and RHIC energies, and a quark-gluon plasma is expected to be created in the early stage of heavy ion collisions. Taking into account the advantage of high luminosity in fixed-target experiments, which is helpful for detailed study of rare particles, the $J / \psi$ yield in $\mathrm{Pb}+\mathrm{Pb}$ collisions at $E_{\text {lab }}=2.76 \mathrm{ATeV}$ per LHC run year is about 100 times larger than the $J / \psi$ yield in $\mathrm{Au}+\mathrm{Au}$ collisions at $\sqrt{s_{\mathrm{NN}}}=62.4 \mathrm{GeV}$ per RHIC run year [1]. With the high statistics, one may precisely distinguish between different cold and hot nuclear matter effects on charmonium production [3]. As is well known, the shadowing effect [4, 5], namely, the difference between the parton distributions in a nucleus and in a free nucleon, depends strongly on the parton momentum fraction $x$. Since $x$ runs in a wide region, $0.001 \leqslant x \leqslant 0.5$, in the fixed-target experiments, it provides a chance to see clearly the shadowing effect on the charmonium distributions in different rapidity bins. In this paper, we study the shadowing effect on the nuclear modification factors for $J / \psi$ yield and transverse momentum in $\mathrm{Pb}+\mathrm{Pb}$ collisions at LHC beam energy $E_{\mathrm{lab}}=2.76 \mathrm{ATeV}$.

\section{Evolution of Quark-Gluon Plasma}

The medium created in heavy ion collision at $\sqrt{s_{\mathrm{NN}}}=$ $72 \mathrm{GeV}$ is assumed to reach local equilibrium at a proper time $\tau_{0}=0.6 \mathrm{fm} / \mathrm{c}[6]$; its consequent space-time evolution can be controlled by the ideal hydrodynamic equations:

$$
\begin{aligned}
\partial_{\mu} T^{\mu \nu} & =0, \\
\partial_{\mu} j^{\mu} & =0,
\end{aligned}
$$

where $T_{\mu \nu}=(\epsilon+p) u_{\mu} u_{\nu}-g_{\mu \nu} p, j_{\mu}=n u_{\mu}$, and $u_{\mu}$, $\epsilon$, $p$, and $n$ are, respectively, the energy-momentum tensor, baryon current, four-velocity of the fluid cell, energy density, pressure, and baryon density of the system. The solution of the hydrodynamic equations provides the local temperature $T(x)$, baryon chemical potential $\mu(x)$, and fluid velocity $u_{\mu}(x)$ of the medium which will be used in the calculation 
of the charmonium suppression and regeneration rates [7]. Taking the assumption of Hubble-like expansion and initial boost invariance along the colliding direction for high energy nuclear collisions, we can employ the well tested $2+1$ dimensional version of the hydrodynamics in describing the evolution of the medium created at $\sqrt{s_{\mathrm{NN}}}=72 \mathrm{GeV}$. Introducing the proper time $\tau=\sqrt{t^{2}-z^{2}}$ and space-time rapidity $\eta=1 / 2 \ln [(t+z) /(t-z)]$ instead of the time $t$ and longitudinal coordinate $z$, the conservation equations can be simplified as [8]

$$
\begin{aligned}
\partial_{\tau} E+\nabla \mathbf{M} & =-\frac{E+p}{\tau}, \\
\partial_{\tau} M_{x}+\nabla\left(M_{x} \mathbf{v}\right) & =-\frac{M_{x}}{\tau}-\partial_{x} p \\
\partial_{\tau} M_{y}+\nabla\left(M_{y} \mathbf{v}\right) & =-\frac{M_{y}}{\tau}-\partial_{y} p \\
\partial_{\tau} R+\nabla(R \mathbf{v}) & =-\frac{R}{\tau}
\end{aligned}
$$

with the definitions $E=(\epsilon+p) \gamma^{2}-p, \mathbf{M}=(\epsilon+p) \gamma^{2} \mathbf{v}$, and $R=\gamma n$, where $\mathbf{v}$ and $\gamma$ are the three-velocity of the fluid cell and Lorentz factor in the transverse plane.

To close the hydrodynamical equations one needs to know the equation of state of the medium. From recent studies on particle elliptic flow and shear viscosity, the matter created in heavy ion collisions at RHIC and LHC energies is very close to a perfect fluid [9]. Considering that the momentum integrated particle yield, especially for heavy quarkonia, is not sensitive to the equation of state, we follow [10] where the deconfined phase at high temperature is an ideal gas of gluons and massless $u$ and $d$ quarks plus $150 \mathrm{MeV}$ massed $s$ quarks, and the hadron phase at low temperature is an ideal gas of all known hadrons and resonances with mass up to $2 \mathrm{GeV}$ [11]. There is a first-order phase transition between these two phases. In the mixed phase, the Maxwell construction is used. The mean field repulsion parameter and the bag constant are chosen as $K=450 \mathrm{MeV} \mathrm{fm}^{3}$ and $B^{1 / 4}=$ $236 \mathrm{MeV}$ to obtain the critical temperature $T_{c}=165 \mathrm{MeV}$ [10] at vanishing baryon number density. Note that when one calculates the rapidity or transverse momentum distribution of quarkonia, the choice of the equation of state may result in sizeable difference.

The initialization of the hot medium is taken as the same treatment in [8]. We use the final charged multiplicity to determine the initial entropy density. For $\sqrt{s_{\mathrm{NN}}}=72 \mathrm{GeV}$, the charged multiplicity at central rapidity in center-of-mass frame is estimated to be $d N_{\mathrm{ch}} / d \eta=515$ based on the empirical formula [12]:

$$
\frac{d N_{\mathrm{ch}}}{d \eta}=312.5 \log _{10} \sqrt{s_{\mathrm{NN}}}-64.8 .
$$

The initial baryon density is obtained by adjusting the entropy per baryon to be 250 [13]. From the empirical relation $\sigma_{\mathrm{NN}}=29.797+0.141\left(\ln \sqrt{s_{\mathrm{NN}}}\right)^{2.624}[14]$ between the inelastic nucleon-nucleon cross section $\sigma_{\mathrm{NN}}$ in unit of $\mathrm{mb}$ and the colliding energy $\sqrt{s_{\mathrm{NN}}}$ in unit of $\mathrm{GeV}$, we have $\sigma_{\mathrm{NN}}=$ $36 \mathrm{mb}$ at $\sqrt{s_{\mathrm{NN}}}=72 \mathrm{GeV}$. These initial conditions lead to a maximum medium temperature $T_{0}=310 \mathrm{MeV}$ at the initial time $\tau_{0}=0.6 \mathrm{fm} / \mathrm{c}$. The medium maintains local chemical and thermal equilibrium during the evolution. If we do not consider the charmonium interaction with the hadron gas, the charmonium distributions in the final state will be fixed at time $\tau_{c}$ corresponding to the critical temperature $T_{c}$ of the deconfinement phase transition.

\section{Charmonium Transport in Quark-Gluon Plasma}

Since a charmonium is so heavy, its equilibrium with the medium can hardly be reached; we use a Boltzmann transport equation to describe its phase space distribution function $f_{\Psi}(x, \mathbf{p} \mid \mathbf{b})$ in heavy ion collisions at impact parameter $\mathbf{b}$ :

$$
p^{\mu} \partial_{\mu} f_{\Psi}=-C_{\Psi} f_{\Psi}+D_{\Psi}
$$

where the loss and gain terms $C_{\Psi}(x, \mathbf{p} \mid \mathbf{b})$ and $D_{\Psi}(x, \mathbf{p} \mid \mathbf{b})$ come from the charmonium dissociation and regeneration in the created hot medium. We have neglected here the elastic scattering, since the charmonium mass is much larger than the typical medium temperature. Considering that the feeddown from the excited states $\psi^{\prime}$ and $\chi_{c}$ to the ground state $J / \psi[15]$ happens after the medium evolution, we should take transport equations for $\Psi=J / \psi, \psi^{\prime}$ and $\chi_{c}$ when we calculate the $J / \psi$ distribution $f_{J / \psi}$ in the final state.

Introducing the momentum rapidity $y=1 / 2 \ln [(E+$ $\left.\left.p_{z}\right) /\left(E-p_{z}\right)\right]$ and transverse energy $E_{t}=\sqrt{E^{2}-p_{z}^{2}}$ to replace the longitudinal momentum $p_{z}$ and energy $E=\sqrt{m^{2}+\mathbf{p}^{2}}$, the transport equation can be rewritten as

$$
\begin{aligned}
& {\left[\cosh (y-\eta) \partial_{\tau}+\frac{\sinh (y-\eta)}{\tau} \partial_{\eta}+\mathbf{v}_{t} \cdot \nabla_{t}\right] f_{\Psi}} \\
& \quad=-\alpha_{\Psi} f_{\Psi}+\beta_{\Psi}
\end{aligned}
$$

with the dissociation and regeneration rates $\alpha_{\Psi}(x, \mathbf{p} \mid \mathbf{b})=$ $C_{\Psi}(x, \mathbf{p} \mid \mathbf{b}) / E_{t}$ and $\beta_{\Psi}(x, \mathbf{p} \mid \mathbf{b})=D_{\Psi}(x, \mathbf{p} \mid \mathbf{b}) / E_{t}$, where the third term in the square bracket arises from the free streaming of $\Psi$ with transverse velocity $\mathbf{v}_{t}=\mathbf{p}_{t} / E_{t}$ which leads to a strong leakage effect at SPS energy [16].

Considering the gluon dissociation $\Psi+g \rightarrow c+\bar{c}$ in the quark-gluon plasma, the dissociation rate $\alpha$ can be expressed as

$$
\begin{aligned}
\alpha_{\Psi} & =\frac{1}{2 E_{t}} \int \frac{d^{3} \mathbf{k}}{(2 \pi)^{3} 2 E_{g}} \sigma_{g \Psi}(\mathbf{p}, \mathbf{k}, T) 4 F_{g \Psi}(\mathbf{p}, \mathbf{k}) \\
& \cdot f_{g}\left(\mathbf{k}, T, u_{\mu}\right),
\end{aligned}
$$

where $E_{g}$ is the gluon energy, $F_{g \Psi}=\sqrt{(p k)^{2}-m_{\Psi}^{2} m_{g}^{2}}=$ $p k$ the flux factor, and $f_{g}$ the gluon thermal distribution as a function of the local temperature $T(x \mid \mathbf{b})$ and fluid velocity $u_{\mu}(x \mid \mathbf{b})$ determined by the hydrodynamics. The dissociation cross section in vacuum $\sigma_{g \Psi}(\mathbf{p}, \mathbf{k}, 0)$ can be 
derived through the operator production expansion (OPE) method with a perturbative Coulomb wave function [1721]. However, the method is no longer valid for loosely bound states at high temperature. To reasonably describe the temperature dependence of the cross section, we take the geometric relation between the averaged charmonium size and the cross section:

$$
\sigma_{g \Psi}(\mathbf{p}, \mathbf{k}, T)=\frac{\left\langle r^{2}\right\rangle_{\Psi}(T)}{\left\langle r^{2}\right\rangle_{\Psi}(0)} \sigma_{g \Psi}(\mathbf{p}, \mathbf{k}, 0) .
$$

The averaged radial square $\left\langle r^{2}\right\rangle_{\Psi}(T)$ is calculated via potential model [22] with lattice simulated heavy quark potential [23] at finite temperature. When $T$ approaches the charmonium dissociation temperature $T_{d}$, the averaged radius square and in turn the cross section go to infinity, which means a complete charmonium melting induced by color screening [24]. Using the internal energy $U$ as the heavy quark potential $V$, the dissociation temperature $T_{d}$ is calculated to be $2.1 T_{c}$, $1.16 T_{c}$, and $1.12 T_{c}$ for $J / \psi, \chi_{c}$, and $\psi^{\prime}$, respectively [22].

The regeneration rate $\beta$ is connected to the dissociation rate $\alpha$ via the detailed balance between the gluon dissociation process and its inverse process $[25,26]$. To obtain the regeneration rate, we also need the charm quark distribution function in medium. Although the initially produced charm quarks would carry high transverse momentum, they lose energy (momentum) when passing through the medium. Considering the experimentally observed large open charm quench factor [27-29] and elliptic flow [30,31], we take as a first approximation a kinetically thermalized momentum spectrum for the charm quark distribution $f_{c}(x, \mathbf{q} \mid \mathbf{b})$. Neglecting the creation and annihilation of charm-anticharm pairs inside the medium, the spatial density of charm quark number $\rho_{c}(x \mid \mathbf{b})=\int d^{3} \mathbf{q} /(2 \pi)^{3} f_{c}(x, \mathbf{q} \mid \mathbf{b})$ satisfies the conservation law

$$
\partial_{\mu}\left(\rho_{c} u^{\mu}\right)=0
$$

with the initial density determined by the nuclear geometry $\rho_{c}\left(x_{0} \mid \mathbf{b}\right)=T_{A}\left(\mathbf{x}_{t}\right) T_{B}\left(\mathbf{x}_{t}-\mathbf{b}\right) \cosh \eta / \tau_{0} d \sigma_{c \bar{c}}^{\mathrm{NN}} / d \eta$, where $T_{A, B}\left(\mathbf{x}_{t}\right)=\int_{-\infty}^{+\infty} \rho_{A, B}(\vec{r}) d z$ are the thickness functions and $d \sigma_{c \bar{c}}^{\mathrm{NN}} / d \eta$ is the charm quark rapidity distribution in $\mathrm{p}+\mathrm{p}$ collisions.

For the regeneration rate $\beta$, we also considered the canonical effect which is shown to be important in explaining the suppression of strange mesons [32]. When there are only few pairs or even less than one pair of charm quarks produced in an event, one needs to consider the canonical effect to guarantee the exact charm number conservation. Taking into account the fact that the charm and anticharm quarks inside a pair are produced at the same rapidity, we simply multiply the regeneration rate $\beta$ in a unit rapidity bin by a canonical enhancement factor [33]

$$
C_{c \bar{c}}=1+\frac{1}{\left(d N_{c \bar{c}} / d y\right)} .
$$

To take into account the relativistic effect on the dissociation cross section to avoid the divergence in the regeneration cross section, we should replace the charmonium binding energy by the gluon threshold energy in the calculations of $\alpha$ and $\beta$ [34].

In the hadron phase of the fireball with temperature $T<T_{c}$, there are many effective models that can be used to calculate the inelastic cross sections between charmonia and hadrons [35]. For $J / \psi$ the dissociation cross section is about a few mb which is comparable with the gluon dissociation cross section. However, considering that the hadron phase appears in the later evolution of the fireball, the ingredient density of the system is much more dilute in comparison with the early hot and dense period [7]. Taking, for instance, the regeneration processes $c+\bar{c} \rightarrow g+J / \psi$ in quark matter and $D+\bar{D}^{*} \rightarrow \pi+J / \psi$ in hadron matter, the density ratio between charm quarks at initial temperature $T_{0}=310 \mathrm{MeV}$ and $D$ mesons at critical temperature $T_{c}=$ $165 \mathrm{MeV}$ is around 30. Considering further the life time of the quark matter $\sim 6 \mathrm{fm} / \mathrm{c}$ and the life time of the hadron matter $\sim 2 \mathrm{fm} / \mathrm{c}$ calculated from the hydrodynamics in Section 2, we neglect the charmonium production and suppression in hadron gas, to simplify the numerical calculations. Note that the suppression and regeneration in hadron gas may become important for excited charmonium states [36].

The transport equation can be solved analytically with the explicit solution $[7,37]$

$$
\begin{aligned}
f_{\Psi} & \left(\mathbf{p}_{t}, y, \mathbf{x}_{t}, \eta, \tau\right)=f_{\Psi}\left(\mathbf{p}_{t}, y, \mathbf{X}_{t}\left(\tau_{0}\right), H\left(\tau_{0}\right), \tau_{0}\right) \\
\cdot & e^{-\int_{\tau_{0}}^{\tau} d \tau^{\prime} / \Delta\left(\tau^{\prime}\right) \alpha_{\Psi}\left(\mathbf{p}_{t}, y, \mathbf{X}_{t}\left(\tau^{\prime}\right), H\left(\tau^{\prime}\right), \tau^{\prime}\right)} \\
+ & \int_{\tau_{0}}^{\tau} \frac{d \tau^{\prime}}{\Delta\left(\tau^{\prime}\right)} \beta_{\Psi}\left(\mathbf{p}_{t}, y, \mathbf{X}_{t}\left(\tau^{\prime}\right), H\left(\tau^{\prime}\right), \tau^{\prime}\right) \\
& \cdot e^{-\int_{\tau^{\prime}}^{\tau} d \tau^{\prime \prime} / \Delta\left(\tau^{\prime \prime}\right) \alpha_{\Psi}\left(\mathbf{p}_{t}, y, \mathbf{X}_{t}\left(\tau^{\prime \prime}\right), H\left(\tau^{\prime \prime}\right), \tau^{\prime \prime}\right)}
\end{aligned}
$$

with

$$
\begin{aligned}
& \mathbf{X}_{t}\left(\tau^{\prime}\right)=\mathbf{x}_{t}-\mathbf{v}_{T}\left[\tau \cosh (y-\eta)-\tau^{\prime} \Delta\left(\tau^{\prime}\right)\right] \\
& H\left(\tau^{\prime}\right)=y-\arcsin \left(\frac{\tau}{\tau^{\prime}} \sinh (y-\eta)\right) \\
& \Delta\left(\tau^{\prime}\right)=\sqrt{1+\left(\frac{\tau}{\tau^{\prime}}\right)^{2} \sinh ^{2}(y-\eta)}
\end{aligned}
$$

The first and second terms on the right-hand side of solution (10) indicate the contributions from the initial production and continuous regeneration, respectively, and both suffer from the gluon dissociation in the medium. Since the regeneration happens in the deconfined phase, the regenerated quarkonia would have probability to be dissociated again by the surrounding gluons. The coordinate shifts $\mathbf{x}_{t} \rightarrow \mathbf{X}_{t}$ and $\eta \rightarrow H$ in solution (10) reflect the leakage effect in the transverse and longitudinal directions.

For fixed-target nuclear collisions at $E_{\text {lab }}=2.76 \mathrm{~A} \mathrm{TeV}$, the collision time for the two $\mathrm{Pb}$ nuclei to pass through each other in the center of mass frame is $2 R_{\mathrm{Pb}} m_{\mathrm{N}} /\left(\sqrt{s_{\mathrm{NN}}} / 2\right) \sim 0.35 \mathrm{fm} / \mathrm{c}$, which is compatible with the charmonium formation time but shorter than the QGP formation time $\tau_{0}=0.6 \mathrm{fm}$. 
Therefore, all the cold nuclear matter effects can be reflected in the initial charmonium distribution $f_{\Psi}$ at time $\tau_{0}$. We take into account nuclear absorption, nuclear shadowing, and Cronin effect. The initial distribution in solution (10) can be obtained from a superposition of $\mathrm{p}+\mathrm{p}$ collisions, along with the modifications from these cold nuclear matter effects.

The nuclear absorption is important in explaining the $J / \psi$ suppression in $\mathrm{p}+\mathrm{A}$ and $\mathrm{A}+\mathrm{A}$ collisions at low energies. It is due to the inelastic collision between the initially produced charmonia and the surrounding nucleons, and its effect on the charmonium surviving probability can be described by an effective absorption cross section $\sigma_{\mathrm{abs}}$. The value of $\sigma_{\mathrm{abs}}$ is usually measured in $\mathrm{p}+\mathrm{A}$ collisions and is several $\mathrm{mb}$ at SPS energy. Since the nuclear absorption becomes weaker at higher colliding energy due to the shorter collision time [38, 39], we take $\sigma_{\mathrm{abs}}=2 \mathrm{mb}$ at $E_{\text {lab }}=2.76 \mathrm{~A} \mathrm{TeV} \mathrm{[39]} \mathrm{and} \mathrm{the}$ nuclear absorption factor

$$
S_{\mathrm{abs}}=e^{-\sigma_{\mathrm{abs}}\left(\int_{z_{A}}^{\infty} \rho\left(z, \mathbf{x}_{t}\right) d z+\int_{-\infty}^{z_{B}} \rho\left(z, \mathbf{x}_{t}-\mathbf{b}\right) d z\right)} .
$$

The Cronin effect broadens the momentum distribution of the initially produced charmonia in heavy ion collisions [7]. In $\mathrm{p}+\mathrm{A}$ and $\mathrm{A}+\mathrm{A}$ collisions, the incoming partons (both gluons and quarks) experience multiple scatterings with surrounding nucleons via soft gluon exchanges. The initial scatterings lead to an additional transverse momentum broadening of partons which is then inherited by produced hadrons [40]. Since the Cronin effect is caused by soft interactions, rigorous calculations for the effect are not available. However, the effect is often treated as a random motion. Inspired from a random-walk picture, we take a Gaussian smearing $[41,42]$ for the modified transverse momentum distribution:

$$
\begin{aligned}
& \bar{f}_{\Psi}^{\mathrm{NN}}\left(\mathbf{x}, \mathbf{p}, z_{A}, z_{B} \mid \mathbf{b}\right) \\
& \quad=\frac{1}{\pi a_{g N} l} \int d^{2} \mathbf{p}_{t}^{\prime} e^{-\mathbf{p}_{t}^{\prime 2} / a_{g N} l} f_{\Psi}^{\mathrm{NN}}\left(\left|\mathbf{p}_{t}-\mathbf{p}_{t}^{\prime}\right|, p_{z}\right) S_{\mathrm{abs}},
\end{aligned}
$$

where

$$
\begin{aligned}
& l\left(\mathbf{x}, z_{A}, z_{B} \mid \mathbf{b}\right) \\
& \quad=\frac{1}{\rho}\left(\int_{-\infty}^{z_{A}} \rho\left(z, \mathbf{x}_{t}\right) d z+\int_{z_{B}}^{+\infty} \rho\left(z, \mathbf{x}_{t}-\mathbf{b}\right) d z\right)
\end{aligned}
$$

is the path length of the two initial gluons in nuclei before fusing into a charmonium at $\mathbf{x}, z_{A}$ and $z_{B}, a_{g N}$ is the averaged charmonium transverse momentum square gained from the gluon scattering with a unit of length of nucleons, and $f_{\Psi}^{\mathrm{NN}}(\mathbf{p})$ is the charmonium momentum distribution in a free $\mathrm{p}+\mathrm{p}$ collision. The Cronin parameter $a_{g N}$ is usually extracted from corresponding $\mathrm{p}+\mathrm{A}$ collisions. Considering the absence of $\mathrm{p}+\mathrm{A}$ collision data at $\sqrt{s_{\mathrm{NN}}}=72 \mathrm{GeV}$, we take $a_{g N}=$ $0.085(\mathrm{GeV} / \mathrm{c})^{2} / \mathrm{fm}$ from some empirical estimations [4, 25, 43]. As a comparison, for collisions at SPS $\left(\sqrt{s_{\mathrm{NN}}} \sim 20 \mathrm{GeV}\right)$ and RHIC $\left(\sqrt{s_{\mathrm{NN}}}=200 \mathrm{GeV}\right)$ we take $a_{g N}=0.075$ [8] and $0.1[44](\mathrm{GeV} / \mathrm{c})^{2} / \mathrm{fm}$, respectively.

Assuming that the emitted gluon in the gluon fusion process $g+g \rightarrow \Psi+g$ is soft in comparison with the initial gluons and the produced charmonium and can be neglected in kinematics, the charmonium production becomes a $2 \rightarrow$ 1 process approximately, and the longitudinal momentum fractions of the two initial gluons are calculated from the momentum conservation:

$$
x_{1,2}=\frac{\sqrt{m_{\Psi}^{2}+p_{t}^{2}}}{\sqrt{s_{\mathrm{NN}}}} e^{ \pm y} .
$$

The free distribution $f_{\Psi}^{\mathrm{NN}}(\mathbf{p})$ can be obtained by integrating the elementary partonic process:

$$
\begin{aligned}
& \frac{d \sigma_{\Psi}^{\mathrm{NN}}}{d p_{t} d y} \\
& \quad=\int d y_{g} x_{1} x_{2} f_{g}\left(x_{1}, \mu_{F}\right) f_{g}\left(x_{2}, \mu_{F}\right) \frac{d \sigma_{g g \rightarrow \Psi g}}{d \widehat{t}},
\end{aligned}
$$

where $f_{g}\left(x, \mu_{F}\right)$ is the gluon distribution in a free proton, $y_{g}$ the emitted gluon rapidity, $d \sigma_{g g \rightarrow \Psi_{g}} / d \widehat{t}$ the charmonium momentum distribution produced from a gluon fusion process, and $\mu_{F}$ the factorization scale of the fusion process.

Now we consider the shadowing effect. The distribution function $\bar{f}_{i}\left(x, \mu_{F}\right)$ for parton $i$ in a nucleus differs from a superposition of the distribution $f_{i}\left(x, \mu_{F}\right)$ in a free nucleon. The nuclear shadowing can be described by the modification factor $R_{i}=\bar{f}_{i} /\left(A f_{i}\right)$. To account for the spatial dependence of the shadowing in a finite nucleus, one assumes that the inhomogeneous shadowing is proportional to the parton path length through the nucleus [45], which amounts to considering the coherent interaction of the incident parton with all the target partons along its path length. Therefore, we replace the homogeneous modification factor $R_{i}\left(x, \mu_{F}\right)$ by an inhomogeneous one [46]

$$
\mathscr{R}_{i}\left(x, \mu_{F}, \mathbf{x}_{t}\right)=1+\frac{A\left(R_{i}\left(x, \mu_{F}\right)-1\right) T_{A}\left(\mathbf{x}_{t}\right)}{T_{A B}(0)}
$$

with the definition $T_{A B}(\mathbf{b})=\int d^{2} \mathbf{x}_{t} T_{A}\left(\mathbf{x}_{t}\right) T_{B}\left(\mathbf{x}_{t}-\mathbf{b}\right)$. We employ in the following the EKS98 package [5] to evaluate the homogeneous ratio $R_{i}$, and the factorization scale is taken as $\mu_{F}=\sqrt{m_{\Psi}^{2}+p_{t}^{2}}$.

Replacing the free distribution $f_{g}$ in (16) by the modified distribution $\bar{f}_{g}=A f_{g} \mathscr{R}_{g}$ and then taking into account the Cronin effect (13), we finally get the initial charmonium distribution for solution (10):

$$
\begin{aligned}
f_{\Psi}\left(x_{0}, \mathbf{p} \mid \mathbf{b}\right)= & \frac{(2 \pi)^{3}}{E_{t} \tau_{0}} \int d z_{A} d z_{B} \rho_{A}\left(\mathbf{x}_{t}, z_{A}\right) \rho_{B}\left(\mathbf{x}_{t}, z_{B}\right) \\
& \cdot \mathscr{R}_{g}\left(x_{1}, \mu_{F}, \mathbf{x}_{t}\right) \mathscr{R}_{g}\left(x_{2}, \mu_{F}, \mathbf{x}_{t}-\mathbf{b}\right) \\
& \cdot \bar{f}_{\Psi}^{\mathrm{NN}}\left(\mathbf{x}, \mathbf{p}, z_{A}, z_{B} \mid \mathbf{b}\right) S_{\mathrm{abs}} .
\end{aligned}
$$

Now the only thing left is the distribution $f_{\Psi}^{\mathrm{NN}}$ in a free $\mathrm{p}+$ $\mathrm{p}$ collision which can be fixed by experimental data or some model simulations. 


\section{Numerical Results}

The beam energy $E_{\mathrm{lab}}=2.76 \mathrm{~A} \mathrm{TeV}$ in fixed-target experiments corresponds to a colliding energy $\sqrt{s_{\mathrm{NN}}}=72 \mathrm{GeV}$, and the rapidity in the center-of-mass frame is boosted in the laboratory frame with a rapidity shift $\Delta y=\tanh ^{-1} \beta_{\mathrm{cms}}=$ 4.3. Let us first focus on the central rapidity region around $y_{\mathrm{cms}}=0$ in the center-of-mass frame, which corresponds to $y_{\text {lab }}=4.3$ in the laboratory frame. The centrality and momentum dependent antishadowing for initially produced charmonia is reflected in the inhomogeneous modification factor $\mathscr{R}_{g}$ for gluons. The longitudinal momentum fractions are $x_{1,2}=\sqrt{m_{\Psi}^{2}+p_{t}^{2}} / \sqrt{s_{\mathrm{NN}}} \sim 0.05$ for the two gluons, which are located at the strong antishadowing region [47] by some parametrization of parton distribution shadowing like EKS98 [5], EPS08 [48], and EPS09 [49]. The antishadowing changes not only the gluon distribution but also the charm quark production cross section used in the regeneration. For the process $g+g \rightarrow c+\bar{c}$, the antishadowing for gluons leads to an antishadowing factor, $\sim\left(\mathscr{R}_{g}\right)^{2}$ for the cross section. Considering that in peripheral collisions the regeneration is weak and its contribution is not remarkably affected by the antishadowing, we take a centrality averaged antishadowing factor for the cross section to simplify the numerical calculation for regeneration. Estimated from the EKS98 evolution [5], we take a $20 \%$ enhancement of the charm quark production cross section compared to free $\mathrm{p}+$ $\mathrm{p}$ collisions. From FONLL calculation [50], the upper limit for $d \sigma_{c \bar{c}}^{\mathrm{NN}} / d y$ is $0.047 \mathrm{mb}$ at $\sqrt{s_{\mathrm{NN}}}=62.4 \mathrm{GeV}$. Note that the experimental data for charm quark cross section in free $\mathrm{p}+\mathrm{p}$ collisions are close to the upper limit of perturbative calculation; we take $d \sigma_{\bar{c}}^{\mathrm{NN}} / d y=0.05 \mathrm{mb}$ at $\sqrt{s_{\mathrm{NN}}}=72 \mathrm{GeV}$. After taking into account the antishadowing effect in $\mathrm{A}+\mathrm{A}$ collisions, it becomes $0.06 \mathrm{mb}$. For $\mathrm{p}+\mathrm{p}$ collisions, we assume a constant hidden to open charm ratio $\left(d \sigma_{\Psi} / d y\right) /\left(d \sigma_{c \bar{c}} / d y\right)=$ const at any colliding energy. From the ratio extracted from the RHIC data [51], we have $d \sigma_{J / \psi} / d y=0.35 \mu \mathrm{b}$ at $\sqrt{s_{\mathrm{NN}}}=$ $72 \mathrm{GeV}$. The transverse momentum distribution for $J / \psi$ in free $\mathrm{p}+\mathrm{p}$ collisions can be simulated by PYTHIA [52] and the mean transverse momentum square is $\left\langle p_{t}^{2}\right\rangle_{\mathrm{pp}}=$ $2.7(\mathrm{GeV} / \mathrm{c})^{2}$.

Figure 1 shows our calculated centrality dependence of $J / \psi$ nuclear modification factor $R_{\mathrm{AA}}=N_{\Psi}^{\mathrm{AA}} /\left(N_{\text {coll }} N_{\Psi}^{\mathrm{pp}}\right)$ in $\mathrm{Pb}$ $+\mathrm{Pb}$ collisions at LHC beam energy $E_{\text {lab }}=2.76 \mathrm{~A} \mathrm{TeV}$ in laboratory frame $\left(\sqrt{s_{\mathrm{NN}}}=72 \mathrm{GeV}\right.$ in center-of-mass frame $)$ at forward rapidity $y_{\text {lab }}=4.3$ (central rapidity $y_{\mathrm{cms}}=0$ ), where $N_{\Psi}^{\mathrm{pp}}$ and $N_{\Psi}^{\mathrm{AA}}$ are charmonium yields in $\mathrm{p}+\mathrm{p}$ and $\mathrm{A}+\mathrm{A}$ collisions, and $N_{\text {coll }}$ and $N_{\text {part }}$ are numbers of binary collisions and participants. For comparison, we show also the RHIC data at $\sqrt{s_{\mathrm{NN}}}=62.4 \mathrm{GeV}$ [53] at central rapidity. Since the shadowing/antishadowing effect is still an open question and its degree depends strongly on the models we used, we show in Figure 1 two calculations for the total $J / \psi R_{\mathrm{AA}}$ in $\mathrm{Pb}+\mathrm{Pb}$ collisions at $\sqrt{s_{\mathrm{NN}}}=72 \mathrm{GeV}$ : one is with the above discussed antishadowing and the other is without antishadowing. The hatched band is due to this uncertainty in the antishadowing. With increasing collision centrality, the initial contribution drops down, while the regeneration goes up. The canonical

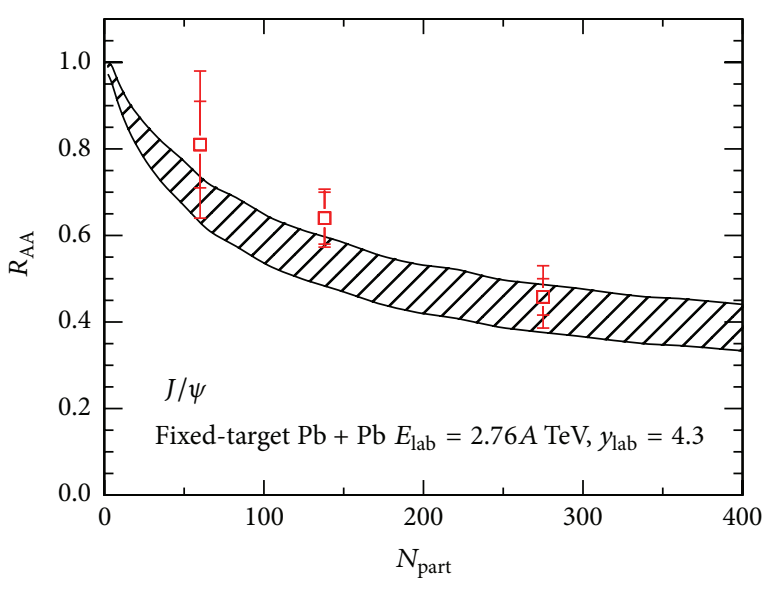

$\square$ STAR data $62.4 \mathrm{GeV}$

FIGURE 1: The centrality dependence of the $J / \psi$ nuclear modification factor $R_{\mathrm{AA}}$ at very forward rapidity $y_{\mathrm{lab}}=4.3\left(y_{\mathrm{cms}}=0\right)$ in $\mathrm{Pb}+\mathrm{Pb}$ collisions at LHC beam energy $E_{\mathrm{lab}}=2.76 \mathrm{~A} \mathrm{TeV}$. The hatched band is the model result with the upper and lower borders corresponding to the calculations with and without antishadowing effect. The RHIC data [53] are for $\mathrm{Au}+\mathrm{Au}$ collisions at $y_{\mathrm{cms}}=0$.

effect is important in peripheral collisions where the number of charm quark pairs is less than one and the inclusion of the canonical effect enhances sizeably the charmonium yield. In most central collisions, the regeneration can contribute about $25 \%$ to the total charmonium yield. The antishadowing at very forward rapidity in the laboratory frame (central rapidity in the center-of-mass frame) enhances the charm quark cross section and in turn the initial charmonium yield by a factor of 1.2. As a consequence, the enhancement factor for the regenerated charmonium number is $1.2^{2}=1.44$ which leads to a strong charmonium enhancement! If we do not consider the antishadowing effect on the charmonium regeneration and initial production, the total $R_{\mathrm{AA}}$ is significantly reduced.

To see more clearly the charmonium production mechanism, we turn to the transverse momentum information. In Figure 2 we show the $J / \psi$ nuclear modification factor [54]

$$
r_{\mathrm{AA}}=\frac{\left\langle p_{t}^{2}\right\rangle_{\mathrm{AA}}}{\left\langle p_{t}^{2}\right\rangle_{\mathrm{pp}}}
$$

in $\mathrm{Pb}+\mathrm{Pb}$ collisions at beam energy $E_{\text {lab }}=2.76 \mathrm{ATeV}$, where $\left\langle p_{t}^{2}\right\rangle_{\mathrm{AA}}$ and $\left\langle p_{t}^{2}\right\rangle_{\mathrm{pp}}$ are averaged $J / \psi$ transverse momentum square in $\mathrm{Pb}+\mathrm{Pb}$ and $\mathrm{p}+\mathrm{p}$ collisions at very forward rapidity $y_{\text {lab }}=4.3$. If we neglect the contribution from the regeneration and consider only the initial production, the ratio $r_{\mathrm{AA}}$ goes up monotonously with centrality due to the Cronin effect and leakage effect [54]. The inclusion of regeneration (upper border of the band) remarkably reduces the averaged transverse momentum, because the regenerated charmonia possess a soft momentum distribution induced by the charm quark energy loss. Since the degree of regeneration increases with centrality, the increased soft component leads to a decreasing $r_{\mathrm{AA}}$ in most central collisions. The canonical effect can reduce the $r_{\mathrm{AA}}$ further, since it enhances the regeneration 


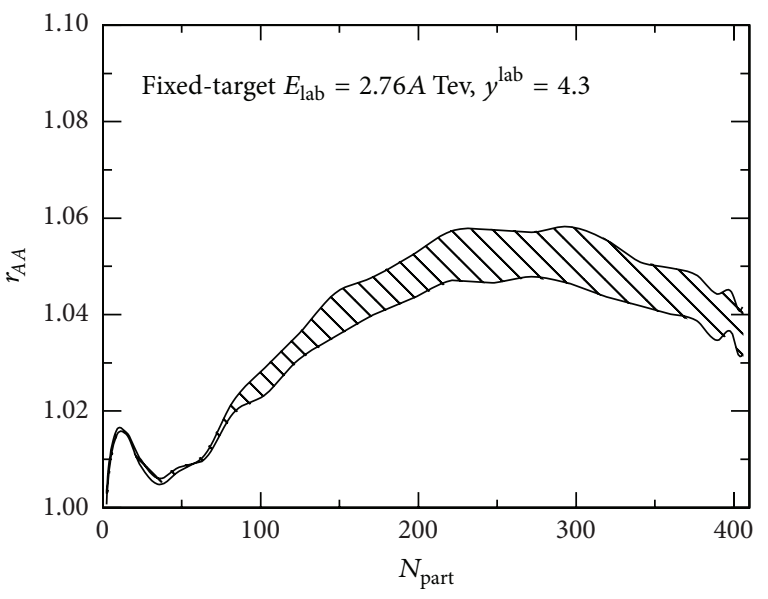

FIGURE 2: The centrality dependence of the $J / \psi$ nuclear modification factor $r_{\mathrm{AA}}$ at forward rapidity $y_{\mathrm{lab}}=4.3\left(y_{\mathrm{cms}}=0\right)$ in $\mathrm{Pb}+\mathrm{Pb}$ collisions at LHC beam energy $E_{\text {lab }}=2.76 A \mathrm{TeV}$. The upper and lower borders of the band correspond to the calculations with and without antishadowing effect.

especially in peripheral collisions. However, we should note that the assumption of charm quark thermalization indicates a full energy loss and it may not be reached in peripheral and semicentral collisions at beam energy $E_{\mathrm{lab}}=2.76 \mathrm{~A} \mathrm{TeV}$. When we switch off the antishadowing (lower border of the band), both the hard component controlled by the initial production and the soft component dominated by the regeneration would be reduced. Considering that the enhancement factor resulted from the antishadowing is 1.2 for the initial production but $1.2^{2}$ for the regeneration, the strong antishadowing in the soft component leads to only a slight difference between with and without considering the antishadowing, shown in Figure 2. It is obvious that, compared to the nuclear modification factor $R_{\mathrm{AA}}$ for the yield, the modification factor $r_{\mathrm{AA}}$ for the transverse momentum is less sensitive to the shadowing effect [54].

From the simulations of parton distributions in cold nuclear matter $[5,48,49]$, the nuclear shadowing region is located at very small $x$. In the following we consider the shadowing and see its difference from the antishadowing in $J / \psi R_{\mathrm{AA}}$ and $r_{\mathrm{AA}}$ in fixed-target $\mathrm{Pb}+\mathrm{Pb}$ collisions. The maximum $J / \psi$ rapidity in the center-of-mass frame is $y_{\mathrm{cms}}^{\max }=\cosh ^{-1}\left[\sqrt{s_{\mathrm{NN}}} /\left(2 m_{J / \psi}\right)\right]=3.13$ at $\sqrt{s_{\mathrm{NN}}}=72 \mathrm{GeV}$. Considering the expected amount of measured events, we focus on the backward rapidity region around $y_{\mathrm{cms}}=-2$ which corresponds to the less forward rapidity $y_{\text {lab }}=\Delta y+$ $y_{\mathrm{cms}}=4.3-2=2.3$ in laboratory frame. From the kinematics, the momentum fractions for the two gluons involved in the gluon fusion process are $x_{1}=\left(\sqrt{m_{\Psi}^{2}+p_{t}^{2}} / \sqrt{s_{\mathrm{NN}}}\right) e^{2}=0.35$ and $x_{2}=\left(\sqrt{m_{\Psi}^{2}+p_{t}^{2}} / \sqrt{s_{\mathrm{NN}}}\right) e^{-2}=0.006$. One is located in the EMC region and the other in the shadowing region $[5,48,49]$, leading to a reduction of $15 \%$ for the charm quark production cross section from EKS98 evolution [5] (20\% from EPS09 NLO evolution [49]). Taking the same ratio of charm quark cross section between $y_{\mathrm{cms}}=-2$ and $y_{\mathrm{cms}}=0$ calculated from

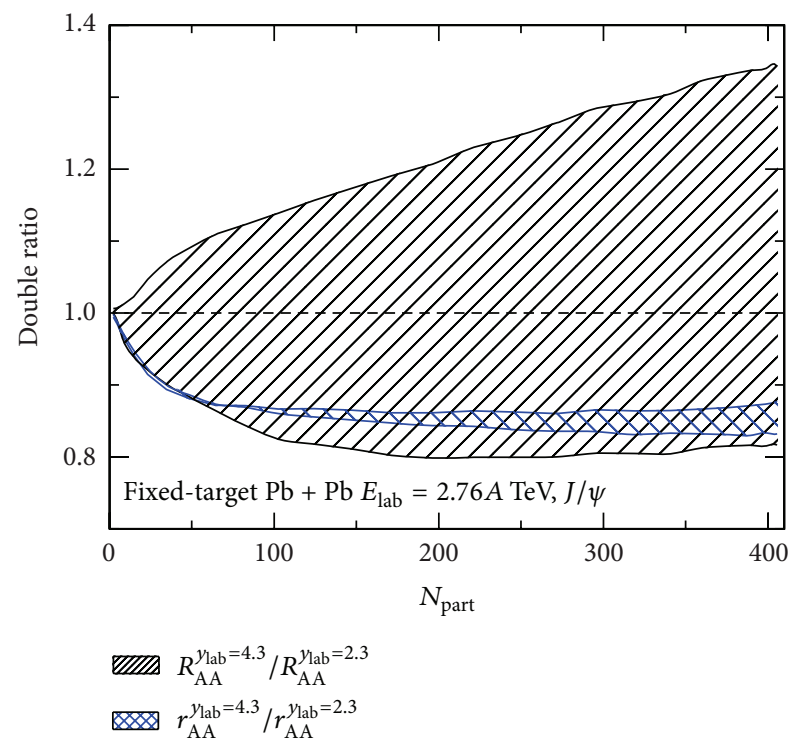

FIgURE 3: The centrality dependence of the double ratios $R_{\mathrm{AA}}^{y_{\mathrm{lab}}=4.3} / R_{\mathrm{AA}}^{y_{\mathrm{lab}}=2.3}$ and $r_{\mathrm{AA}}^{y_{\mathrm{lab}}=4.3} / r_{\mathrm{AA}}^{y_{\text {lab }}=2.3}$ for $J / \psi$ yield and transverse momentum in $\mathrm{Pb}+\mathrm{Pb}$ collisions at LHC beam energy $E_{\text {lab }}=2.76 A \mathrm{TeV}$. The upper and lower borders of the two bands correspond to the calculations with and without shadowing and antishadowing effects.

FONLL [50] and including the $15 \%$ shadowing reduction, we obtain $d \sigma_{c \bar{c}}^{\mathrm{NN}} / d y=0.01 \mathrm{mb}$ at $y_{\mathrm{cms}}=-2$. For the medium evolution at this backward rapidity region, we initialize the entropy density to be half of that at central rapidity $[6,55]$ which leads to a maximum temperature of $T_{0}=245 \mathrm{MeV}$. Figure 3 shows the two double ratios $R_{\mathrm{AA}}^{y_{\mathrm{lab}}=4.3} / R_{\mathrm{AA}}^{y_{\mathrm{lab}}=2.3}$ and $r_{\mathrm{AA}}^{y_{\mathrm{aab}}=4.3} / r_{\mathrm{AA}}^{y_{\mathrm{lab}}=2.3}$ of $J / \psi$; the upper and lower borders of the two bands correspond to the calculations with and without considering the nuclear shadowing and antishadowing. While the double ratio for the transverse momentum is not sensitive to the shadowing and antishadowing, as we discussed above, the strong antishadowing at $y_{\mathrm{lab}}=4.3$ and shadowing at $y_{\mathrm{lab}}=2.3$ lead to a strong enhancement of the double ratio for the yield. Without considering the shadowing and antishadowing, the stronger charmonium suppression in the hotter medium at $y_{\text {lab }}=4.3\left(T_{0}=310 \mathrm{MeV}\right)$ compared with the weaker suppression in the relatively colder medium at $y_{\text {lab }}=2.3\left(T_{0}=245 \mathrm{MeV}\right)$ makes the double ratio less than unit. However, the inclusion of the yield enhancement due to the antishadowing at $y_{\mathrm{lab}}=4.3$ and the yield suppression due to the shadowing at $y_{\mathrm{lab}}=2.3$ changes significantly the behavior of the double rati; it becomes larger than unit and can reach 1.3 in most central collisions. Note that the rapidity dependent shadowing effect was used to qualitatively interpret the stronger suppression at forward rapidity than that at midrapidity in Au + Au collisions at RHIC $[56,57]$.

\section{Summary}

We investigated with a transport approach the charmonium production in fixed-target $\mathrm{Pb}+\mathrm{Pb}$ collisions at $\mathrm{LHC}$ beam 
energy $E_{\text {lab }}=2.76 A \mathrm{TeV}$. We focused on the rapidity dependent shadowing effect on the nuclear modification factors for the charmonium yield and transverse momentum. While the averaged transverse momentum is not sensitive to the shadowing effect, the antishadowing leads to a strong yield enhancement at very forward rapidity $y_{\text {lab }} \simeq 4$, and the shadowing results in a strong yield suppression at less forward rapidity $y_{\text {lab }} \simeq 2$. The double ratio between the nuclear modification factors $R_{\mathrm{AA}}$ in the two rapidity regions amplifies the shadowing effect; it is larger than unit and can reach 1.3 in most central collisions.

From the model studies on gluon distribution in nuclei, see, for instance [5, 47-49], there are large uncertainties in the domain of large $x(>0.1)$, which is probably due to the unknown EMC effect. From our calculation here, the double ratio of the nuclear modification factor for $J / \psi$ yield is very sensitive to the gluon shadowing effect in different $x$ region. A precise measurement of the ratio may provide a sensitive probe to the gluon distribution.

\section{Conflict of Interests}

The authors declare that there is no conflict of interests regarding the publication of this paper.

\section{Acknowledgments}

The work is supported by the NSFC under Grant no. 11335005 and the MOST under Grant nos. 2013 CB922000 and 2014 CB845400.

\section{References}

[1] S. J. Brodsky, F. Fleuret, C. Hadjidakis, and J. P. Lansberg, "Physics opportunities of a fixed-target experiment using LHC beams," Physics Reports, vol. 522, no. 4, pp. 239-255, 2013.

[2] J. P. Lansberg, S. J. Brodsky, F. Fleuret, and C. Hadjidakis, "Quarkonium physics at a fixed-target experiment using the LHC beams," Few-Body Systems, vol. 53, no. 1-2, pp. 11-25, 2012.

[3] A. Andronic, F. Arleo, R. Arnaldi et al., "Heavy-flavour and quarkonium production in the LHC era: from proton-proton to heavy-ion collisions," http://arxiv.org/abs/1506.03981.

[4] R. Vogt, "The A-dependence of open charm and bottom production," International Journal of Modern Physics E, vol. 12, no. 2, p. 211, 2003.

[5] K. J. Eskola, V. J. Kolhinen, and C. A. Salgado, "The scale dependent nuclear effects in parton distributions for practical applications," The European Physical Journal C, vol. 9, no. 1, pp. 61-68, 1999.

[6] C. Shen and U. Heinz, "Collision energy dependence of viscous hydrodynamic flow in relativistic heavy-ion collisions," Physical Review C, vol. 85, no. 5, Article ID 054902, 12 pages, 2012.

[7] Z. Tang, N. Xu, K. Zhou, and P. Zhuang, "Charmonium transverse momentum distribution in high energy nuclear collisions," Journal of Physics G: Nuclear and Particle Physics, vol. 41, no. 12, Article ID 124006, 2014.

[8] X. Zhu, P. Zhuang, and N. Xu, " J/ $\psi$ transport in QGP and $\mathrm{p}_{\mathrm{t}}$ distribution at SPS and RHIC," Physics Letters B, vol. 607, no. 1-2, pp. 107-114, 2005.
[9] H. Song, S. Bass, U. Heinz, T. Hirano, and C. Shen, "200 A GeV $\mathrm{Au}+\mathrm{Au}$ collisions serve a nearly perfect Quark-Gluon liquid," Physical Review Letters, vol. 106, Article ID 192301, 2012.

[10] J. Sollfrank, P. Huovinen, M. Kataja, P. V. Ruuskanen, M. Prakash, and R. Venugopalan, "Hydrodynamical description of $200 \mathrm{~A} \mathrm{GeV/c} \mathrm{S+Au} \mathrm{collisions:} \mathrm{hadron} \mathrm{and} \mathrm{electromagnetic}$ spectra," Physical Review C, vol. 55, article 392, 1997.

[11] K. Hagiwara, K. Hikasa, K. Nakamura et al., "Review of particle properties," Physical Review D, vol. 66, no. 1, Article ID 010001, 2002.

[12] G. Kestin and U. Heinz, "Hydrodynamic radial and elliptic flow in heavy-ion collisions from AGS to LHC energies," The European Physical Journal C, vol. 61, no. 4, pp. 545-552, 2009.

[13] P. Kolb and R. Rapp, "Transverse flow and hadrochemistry in $\mathrm{Au}+\mathrm{Au}$ collisions at $\sqrt{s_{\mathrm{NN}}}=200 \mathrm{GeV}$," Physical Review C, vol. 67, Article ID 044903, 2003.

[14] Particle Data Group, "Review of particle properties," Physical Review D, vol. 45, no. 11, pp. S1-S574, 1992.

[15] A. Zoccoli, I. Abt, M. Adams et al., "Charm, beauty and charmonium production at HERA-B," The European Physical Journal C, vol. 43, no. 1-4, pp. 179-186, 2005.

[16] J. Hüfner and P. Zhuang, "Time structure of anomalous $J / \Psi$ and $\Psi^{\prime}$ suppression in nuclear collisions," Physics Letters B, vol. 559, no. 3-4, pp. 193-200, 2003.

[17] G. Bhanot and M. E. Peskin, "Short-distance analysis for heavyquark systems: (I). Diagrammatics," Nuclear Physics B, vol. 156, no. 3, pp. 365-390, 1979.

[18] G. Bhanot and M. E. Peskin, "Short-distance analysis for heavyquark systems: (II). Applications," Nuclear Physics B, vol. 156, no. 3, pp. 391-416, 1979.

[19] F. Arleo, P.-B. Gossiaux, T. Gousset, and J. Aichelin, "Heavyquarkonium hadron cross section in QCD at leading twist," Physical Review D, vol. 65, no. 1, Article ID 014005, 2002.

[20] Y. S. Oh, H. C. Kim, and S. H. Lee, "Quarkonium-hadron interactions in QCD," Physical Review C, vol. 65, Article ID 067901, 2002.

[21] X. N. Wang, "Azimuthal asymmetry of $J / \psi$ suppression in noncentral heavy-ion collisions," Physics Letters B, vol. 540, no. 1-2, pp. 62-67, 2002.

[22] H. Satz, "Colour deconfinement and quarkonium binding," Journal of Physics G, vol. 32, no. 3, p. R25, 2006.

[23] P. Petreczky, "Quarkonium in a hot medium," Journal of Physics G: Nuclear and Particle Physics, vol. 37, no. 9, Article ID 094009, 2010.

[24] T. Matsui and H. Satz, "J/ $\psi$ suppression by quark-gluon plasma formation," Physics Letters B, vol. 178, pp. 416-422, 1986.

[25] R. L. Thews and M. L. Mangano, "Momentum spectra of charmonium produced in a quark-gluon plasma," Physical Review C, vol. 73, Article ID 014904, 2006.

[26] L. Yan, P. Zhuang, and N. Xu, "J/ $\psi$ production in quark-gluon plasma," Physical Review Letters, vol. 97, Article ID 232301, 2006.

[27] B. I. Abelev, M. M. Aggarwal, Z. Ahammed et al., "Transverse momentum and centrality dependence of high- $p_{T}$ nonphotonic electron suppression in $\mathrm{Au}+\mathrm{Au}$ collisions at $\sqrt{s_{\mathrm{NN}}}=200 \mathrm{GeV}$," Physical Review Letters, vol. 98, Article ID 192301, 2007.

[28] L. Adamczyk, J. K. Adkins, G. Agakishiev et al., "Observation of $D^{0}$ Meson nuclear modifications in Au+Au collisions at $\sqrt{s_{\mathrm{NN}}}=$ 200 GeV," Physical Review Letters, vol. 113, no. 14, Article ID 142301, 7 pages, 2014. 
[29] B. Abelev, J. Adam, D. Adamová et al., "Suppression of high transverse momentum $\mathrm{D}$ mesons in central $\mathrm{Pb}-\mathrm{Pb}$ collisions at $\sqrt{s_{N N}}=2.76$ TeV," Journal of High Energy Physics, vol. 2012, no. 9, article 112, 2012.

[30] A. Adare, S. Afanasiev, C. Aidala et al., "Energy loss and flow of heavy quarks in Au+Au collisions at $\sqrt{s_{\mathrm{NN}}}=200 \mathrm{GeV}$," Physical Review Letters, vol. 98, Article ID 172301, 2007.

[31] B. Abelev, J. Adam, D. Adamová et al., " $D$ meson elliptic flow in noncentral $\mathrm{Pb}-\mathrm{Pb}$ collisions at $\sqrt{s_{N N}}=2.76 \mathrm{TeV}$," Physical Review Letters, vol. 111, Article ID 102301, 2013.

[32] C. M. Ko, V. Koch, Z.-W. Lin, K. Redlich, M. Stephanov, and X.-N. Wang, "Kinetic equation with exact charge conservation," Physical Review Letters, vol. 86, pp. 5438-5441, 2001.

[33] Y. Liu, C. Ko, and T. Song, "Hot medium effects on $J / \psi$ production in $\mathrm{p}+\mathrm{Pb}$ collisions at $\sqrt{s_{\mathrm{NN}}}=5.02$," Physics Letters $B$, vol. 728, pp. 437-442, 2014.

[34] A. Polleri, T. Renk, R. Schneider, and W. Weise, "Kinetic description of charmonium production in high-energy nuclear collisions," Physical Review C, vol. 70, Article ID 044906, 2004.

[35] T. Barnes, E. S. Swanson, C.-Y. Wong, and X.-M. Xu, "Dissociation cross sections of ground state and excited charmonia with light mesons in the quark model," Physical Review C, vol. 68, Article ID 014903, 2003.

[36] X. Du and R. Rapp, "Sequential regeneration of charmonia in heavy-ion collisions," http://arxiv.org/abs/1504.00670.

[37] Y. Liu, Z. Qu, N. Xu, and P. Zhuang, "Rapidity dependence of $J / \psi$ production at the RHIC and LHC," Journal of Physics G: Nuclear and Particle Physics, vol. 37, no. 7, Article ID 075110, 2010.

[38] A. Capella and E. G. Ferreiro, " $J / \psi$ Suppression and the decrease of nuclear absorption with increasing energy," Physical Review C, vol. 76, Article ID 064906, 2007.

[39] C. Lourenço, R. Vogt, and H. K. Wöhri, "Energy dependence of $J / \psi$ absorption in proton-nucleus collisions," Journal of High Energy Physics, vol. 2009, no. 2, article 014, 2009.

[40] S. Esumi, U. Heinz, and N. Xu, "Collective flow or random walk?” Physics Letters B, vol. 403, no. 1-2, pp. 145-148, 1997.

[41] X. Zhao and R. Rapp, "Transverse momentum spectra of $J / \psi$ in heavy-ion collisions," Physics Letters B, vol. 664, no. 4-5, pp. 253-257, 2008.

[42] Y. Liu, B. Chen, N. Xu, and P. Zhuang, "Y production as a probe for early state dynamics in high energy nuclear collisions at RHIC," Physics Letters B, vol. 697, no. 1, pp. 32-36, 2011.

[43] X. N. Wang, "Where is the jet quenching in $P b+P b$ collisions at 158 A GeV?" Physical Review Letters, vol. 81, pp. 2655-2658, 1998.

[44] Y. Liu, Z. Qu, N. Xu, and P. Zhuang, "Spacetime evolution of $J / \psi$ production in high energy nuclear collisions," Journal of Physics $G$, vol. 36, no. 6, Article ID 064057, 2009.

[45] S. R. Klein and R. Vogt, "Inhomogeneous shadowing effects on $J / \psi$ production in $d A$ collisions," Physical Review Letters, vol. 91, Article ID 142301, 2003.

[46] R. Vogt, "Shadowing and absorption effects on $J / \psi$ production in $d A$ collisions," Physical Review C, vol. 71, Article ID 054902, 2005.

[47] J. Dias de Deus, "Nuclear anti-shadowing and the quarkonium nucleon elastic scattering amplitude," Physics Letters B, vol. 335, no. 2, pp. 188-191, 1994.

[48] K. Eskola, H. Paukkunen, and C. Salgado, "An improved global analysis of nuclear parton distribution functions including
RHIC data," Journal of High Energy Physics, vol. 2008, no. 7, article 102, 2008.

[49] K. J. Eskola, H. Paukkunen, and C. A. Salgado, "EPS09-a new generation of NLO and LO nuclear parton distribution functions," Journal of High Energy Physics, vol. 2009, no. 4, article 065, 2009.

[50] M. Cacciari, M. Greco, and P. Nason, "The $p_{T}$ spectrum in heavy-flavour hadroproduction," Journal of High Energy Physics, vol. 1998, no. 5, article 007, 1998.

[51] A. Adare, S. Afanasiev, C. Aidala et al., " $J / \psi$ production versus centrality, transverse momentum, and rapidity in $\mathrm{Au}+\mathrm{Au}$ collisions at $\sqrt{s_{N N}}=200 \mathrm{GeV}$,' Physical Review Letters, vol. 98, Article ID 232301, 2007.

[52] T. Sjöstrand, P. Edén, C. Friberg et al., "High-energy-physics event generation with $\mathrm{P}_{\mathrm{YTHIA}}$ 6.1," Computer Physics Communications, vol. 135, no. 2, pp. 238-259, 2001.

[53] A. Adare, C. Aidala, N. N. Ajitanand et al., " $J / \psi$ suppression at forward rapidity in $\mathrm{Au}+\mathrm{Au}$ collisions at $\sqrt{s_{N N}}=39$ and 62.4 GeV," Physical Review C, vol. 86, Article ID 064901, 2012.

[54] K. Zhou, N. Xu, Z. Xu, and P. Zhuang, "Medium effects on charmonium production at ultrarelativistic energies available at the CERN Large Hadron Collider," Physical Review C, vol. 89, Article ID 054911, 2014.

[55] M. C. Abreu, B. Alessandro, C. Alexa et al., "Scaling of charged particle multiplicity in $\mathrm{Pb}-\mathrm{Pb}$ collisions at SPS energies," Physics Letters B, vol. 530, no. 1-4, pp. 43-55, 2002.

[56] A. D. Frawley, T. Ullrich, and R. Vogt, "Heavy flavor in heavyion collisions at RHIC and RHIC II," Physics Reports, vol. 462, no. 4-6, pp. 125-175, 2008.

[57] E. Ferreiro, F. Fleuret, J. Lansberg, and A. Rakotozafindrabe, "Cold nuclear matter effects on $J / \psi$ production: intrinsic and extrinsic transverse momentum effects," Physics Letters B, vol. 680, pp. 50-55, 2009. 

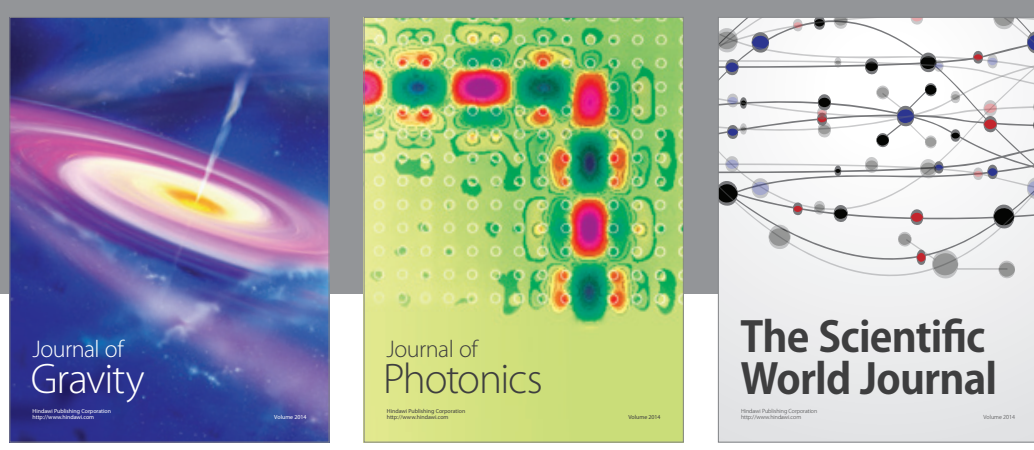

The Scientific World Journal
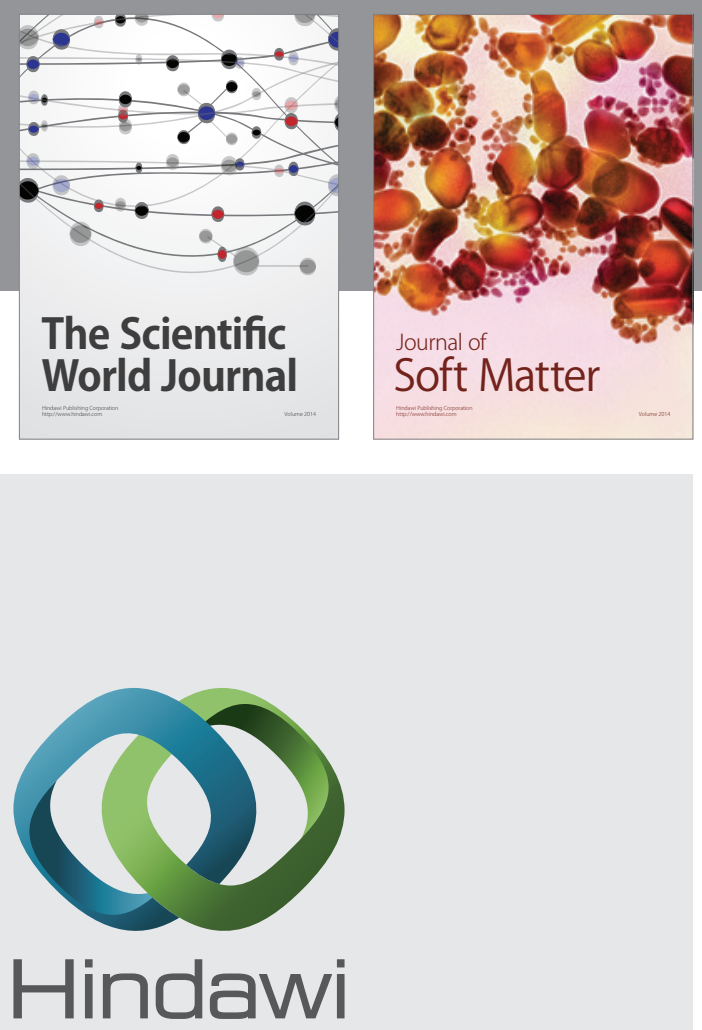

Submit your manuscripts at

http://www.hindawi.com

nternational Journal of

Statistical Mechanics
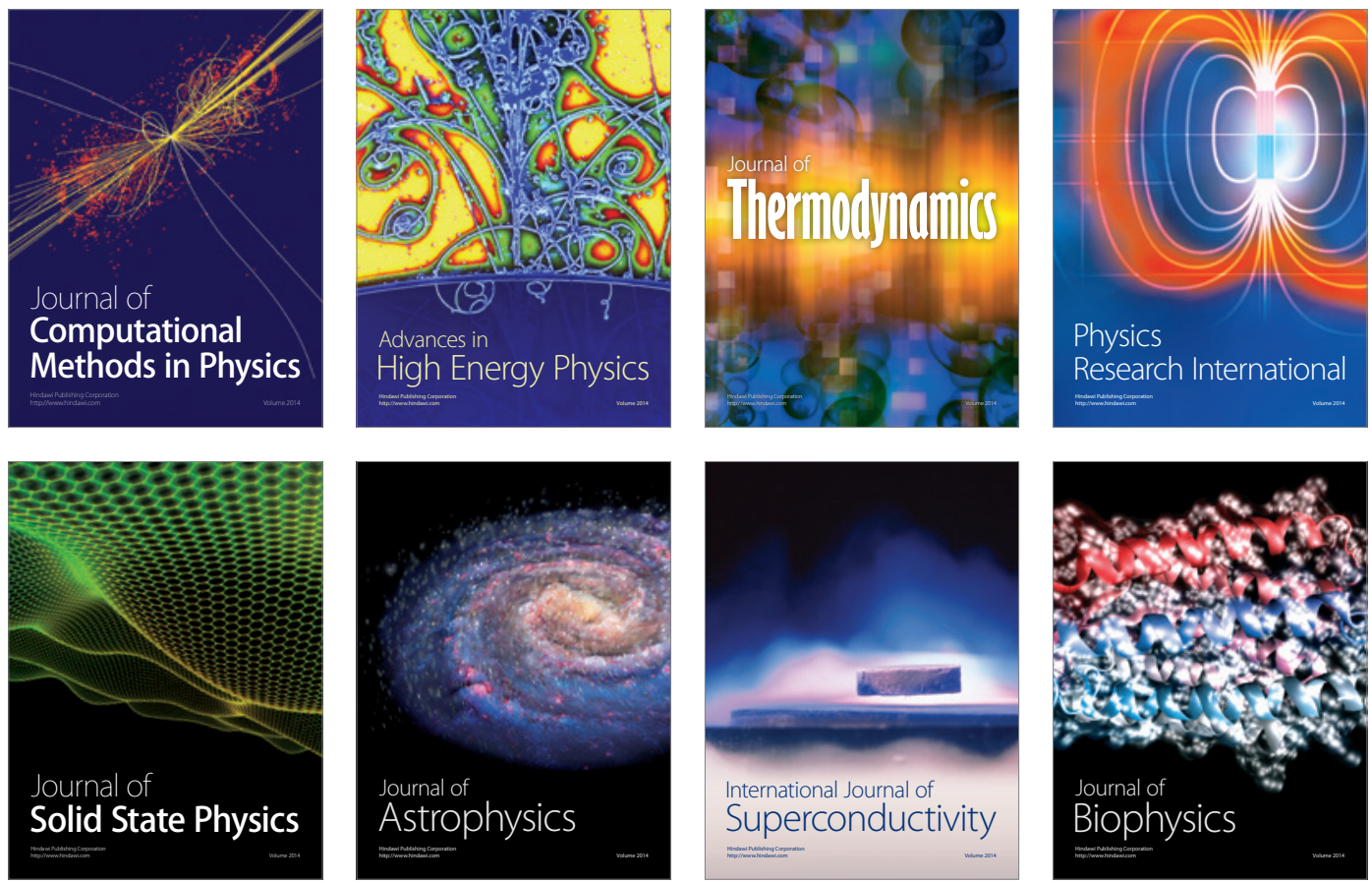
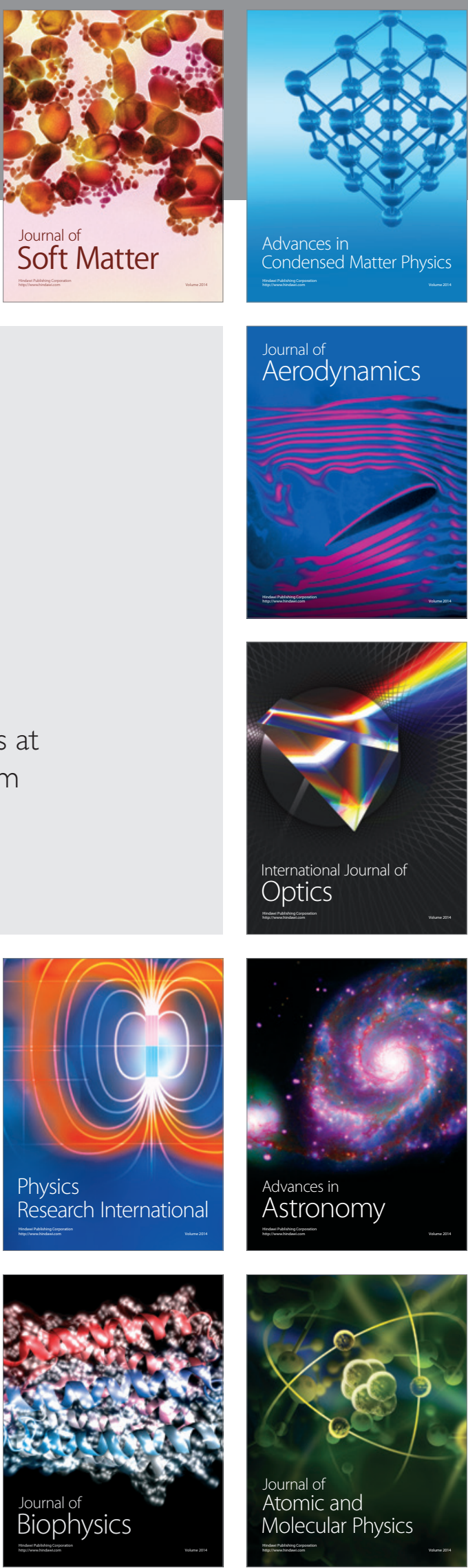